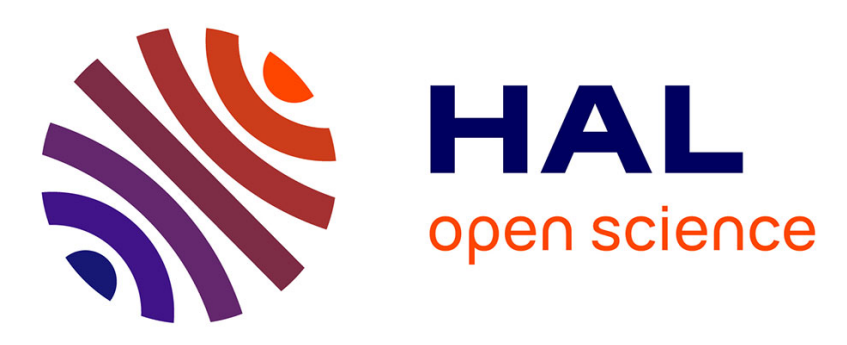

\title{
Rains of solitons in a figure-of-eight passively mode-locked fiber laser
}

Alioune Niang, Foued Amrani, Mohamed Salhi, Philippe Grelu, François

Sanchez

\section{- To cite this version:}

Alioune Niang, Foued Amrani, Mohamed Salhi, Philippe Grelu, François Sanchez. Rains of solitons in a figure-of-eight passively mode-locked fiber laser. Applied Physics B - Laser and Optics, 2014, 116 (3), pp.771-775. 10.1007/s00340-014-5760-y . hal-03204336

\section{HAL Id: hal-03204336 \\ https://univ-angers.hal.science/hal-03204336}

Submitted on 21 Apr 2021

HAL is a multi-disciplinary open access archive for the deposit and dissemination of scientific research documents, whether they are published or not. The documents may come from teaching and research institutions in France or abroad, or from public or private research centers.
L'archive ouverte pluridisciplinaire HAL, est destinée au dépôt et à la diffusion de documents scientifiques de niveau recherche, publiés ou non, émanant des établissements d'enseignement et de recherche français ou étrangers, des laboratoires publics ou privés. 


\title{
Rains of solitons in a figure-of-eight passively mode-locked fiber laser
}

\author{
Alioune Niang • Foued Amrani - Mohamed Salhi • \\ Philippe Grelu $\cdot$ François Sanchez
}

Received: 15 July 2013/ Accepted: 3 January 2014/Published online: 12 January 2014

(C) Springer-Verlag Berlin Heidelberg 2014

\begin{abstract}
We report experimental observation of rains of solitons in figure-of-eight fiber laser passively mode-locked through nonlinear optical loop mirror. Soliton pulses are created from an extended noisy background and drift until they reach a condensed phase comprising several tens of aggregated solitons. The observation of this dynamics tends to strengthen the idea of the universality of the collective behavior of solitons.
\end{abstract}

\section{Introduction}

Passively mode-locked fiber lasers constitute ideal tools for the investigation of nonlinear dynamics in dissipative systems [1-6]. Indeed, they offer many possibilities for the realization of the mode-locking such as nonlinear polarization rotation technique (NLPR) [7-10], figure-of-eight lasers (F8L) configurations [11-13] or a saturable absorbers [3]. In addition, the inhomogeneous broadening resulting from the host material leads to large spectral gain bandwidths which are very favorable for mode-locking applications. In the anomalous dispersion regime, the

Electronic supplementary material The online version of this article (doi:10.1007/s00340-014-5760-y) contains supplementary material, which is available to authorized users.

A. Niang · F. Amrani · M. Salhi · F. Sanchez $(\bowtie)$

Laboratoire de Photonique d'Angers EA 4464, Université

d'Angers, 2 Bd Lavoisier, 49045 Angers Cedex 01, France

e-mail: francois.sanchez@univ-angers.fr

P. Grelu

Laboratoire Interdisciplinaire Carnot de Bourgogne, UMR 5209

CNRS, Université de Bourgogne, B.P. 47870,

21078 Dijon Cedex, France energy of the pulses is quantified and, in general, several solitons coexist in the cavity. The scaling up of the output power of fiber lasers in recent years has allowed a considerable increase in the number of coexisting solitons in passively mode-locked fiber lasers. Thus, the number of interacting pulses has undergone a big step from a few tens to several hundreds. As a consequence of the soliton interactions, different complex patterns can be observed [4, $5,13,14]$. Depending on the nature and on the range of the interaction, soliton distributions can exhibit some order at different scales or can be completely disordered. For lowpower fiber lasers, the formation of bound states including few pulses has been widely studied both theoretically and experimentally leading to the emergence of the dissipative soliton dynamics [15-18]. While the average power in fiber lasers has increased, the resulting large number of interacting solitons yields to complex temporal distributions. A first attempt to give a physical classification of the different patterns has been done [4] in the case of the Er-doped double-clad fiber laser passively mode-locked through NLPR. The authors have pointed out a formal analogy between the soliton distributions and the different states of the matter. Additional physical insight on soliton temporal distribution was obtained thanks to a reconstruction of the different patterns [14]. Similar soliton patterns have been reported in the figure-of-eight double-clad fiber laser mode-locked through nonlinear amplifying loop mirror (NALM) [13]. Such results strongly suggest that the soliton distributions observed in fiber lasers operating in the anomalous regime have some universal character. Indeed, soliton patterns do not seem to depend on the exact modelocking mechanism. At intermediate power level, it has been reported a rain of solitons in the anomalous dispersion Er-doped fiber laser passively mode-locked through NLPR [19]. This dynamical behavior appears when there is 
coexistence of a soliton cluster and a noisy quasi-cw background. When the background level is sufficiently high, solitons emerge spontaneously and randomly from the background fluctuations, then they drift to the condensate phase. The relative velocity of the individual solitons versus the condensed phase remains constant. The background fluctuations are due to a large number of noncoherent oscillation modes. On the other hand, Bao et al. [20] have recently observed soliton rains in normal dispersion Yb-fiber laser with dual-filter. However, soliton rain was relatively difficult to obtain because the pulse propagation in normal dispersion can tolerate larger nonlinear phase shifts.

The aim of this paper is to investigate the soliton patterns formation in the F8L using the nonlinear optical loop mirror (NOLM) configuration. We have experimentally investigated soliton dynamics with cavity parameters as well as the pumping power and the polarization controllers. Different soliton complexes have been obtained, but we focus here on the rain of solitons which has never been observed in F8L.

\section{Experimental results}

The experimental setup is schematically represented in Fig. 1. It is an all-fiber figure-of-eight laser based on a passive unidirectional ring (UR) cavity which is coupled to a NOLM through a 80/20 fiber coupler. The unidirectional ring (UR) part is composed of an optical isolator, a $10 \%$ output coupler and contains a double-clad 1-W Er/Ybdoped fiber amplifier manufactured by Keopsys. The latter consists in a 8-m-long double-clad fiber (DCF) that has a chromatic dispersion coefficient $\beta_{2}=0.015 \mathrm{ps}^{2} / \mathrm{m}$ at the operating wavelength $1550 \mathrm{~nm}$. The v-groove technique is used to launch the pump beams into the inner cladding. Two identical laser diodes operating at $980 \mathrm{~nm}$ and each emitting about $3 \mathrm{~W}$ are used in a counter-propagating geometry. Mode-locking is obtained through the adjustment of the polarization controller (PC) placed in the NOLM. The total dispersion is set in the anomalous regime with $\beta_{2}^{\text {TOT }} L=-0.54 \mathrm{ps}^{2}$, with a total cavity length of $28.1 \mathrm{~m}$ corresponding to a round-trip time of $139.6 \mathrm{~ns}$. A $10 \%$ output coupler, placed just after the amplifier, is used to extract the power from the cavity. The output beam is detected with a high-speed photodiode (Newport TIA 1200 $13 \mathrm{GHz}$ ) and analyzed with a high-speed oscilloscope (Tektronix TDS 6124C $12 \mathrm{GHz}, 40 \mathrm{GS} / \mathrm{s}$ ). An optical spectrum analyzer (Anritsu MS 9710C) is used to observe the spectral response of the laser when needed. The pulse duration is measured with an optical autocorrelator (Femtochrome FR-103 XL) with a scanning range of about \pm 100 ps.

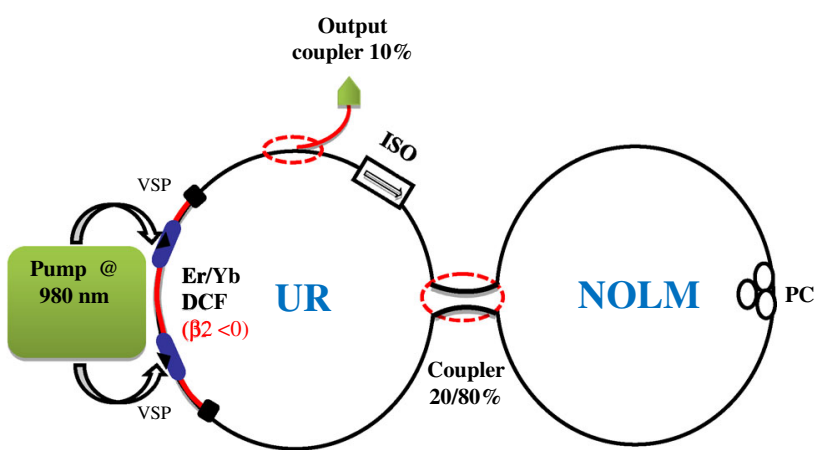

Fig. 1 Experimental setup. DCF double-clad fiber, ISO optical isolator, $P C$ polarization controller, NOLM nonlinear optical loop mirror, $U R$ unidirectional ring controller

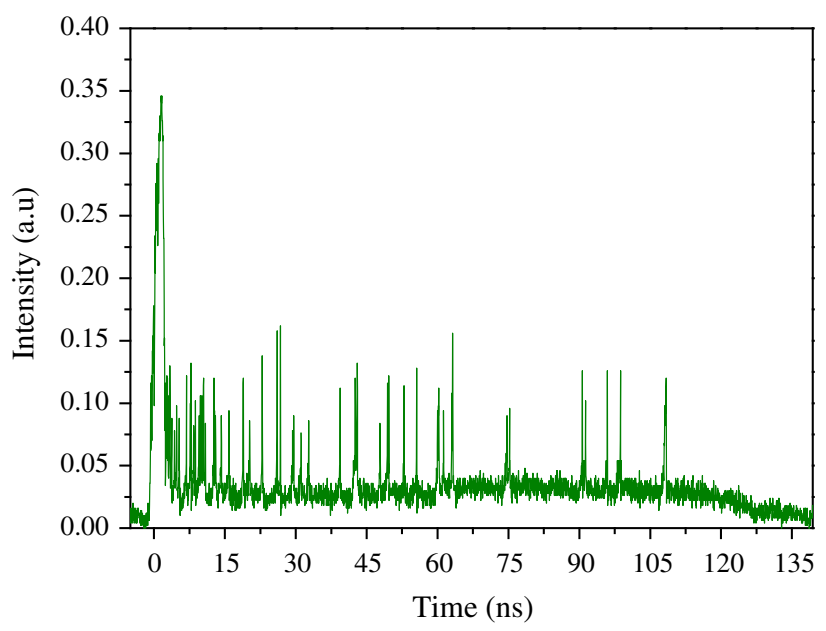

Fig. 2 Temporal trace of a solitons rains

For a pump power of about $3 \mathrm{~W}$ and a proper adjustment of polarization controllers, we obtain the temporal distribution given in Fig. 2. It consists in a condensate solitons state with a large tail which is a quasi-cw background. The condensed state span over about 4 ns with a separation between solitons of approximately $180 \mathrm{ps}$ (this value is an average value obtained from data series delivered by the fast oscilloscope in the limit of its temporal resolution which does not allow to distinguish between two pulses closer that $75 \mathrm{ps}$ ). From these two values, we estimate the number of pulses within the condensed phase to be about 20. The temporal extension of the packet of solitons undergoes small fluctuations and depends on both the pump power and the nonlinear filtering as will be shown later. This regime repeats with a period equals to the round-trip time of the cavity. However, the distribution of the pulses is not at rest, but instead several isolated solitons appear spontaneously and randomly from background fluctuations and then drift (right to left) to the condensed phase and merge with it. This video (click here to see the attached 


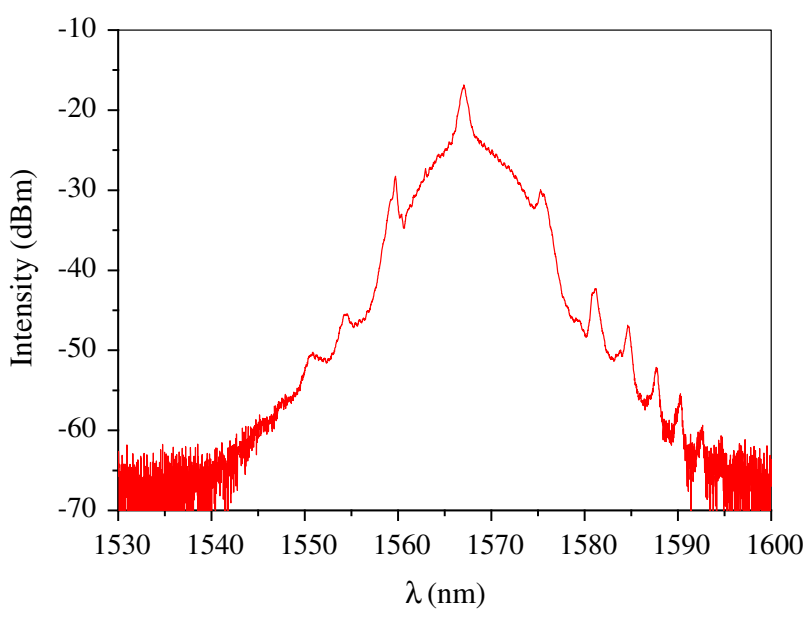

Fig. 3 Optical spectrum of a solitons rain

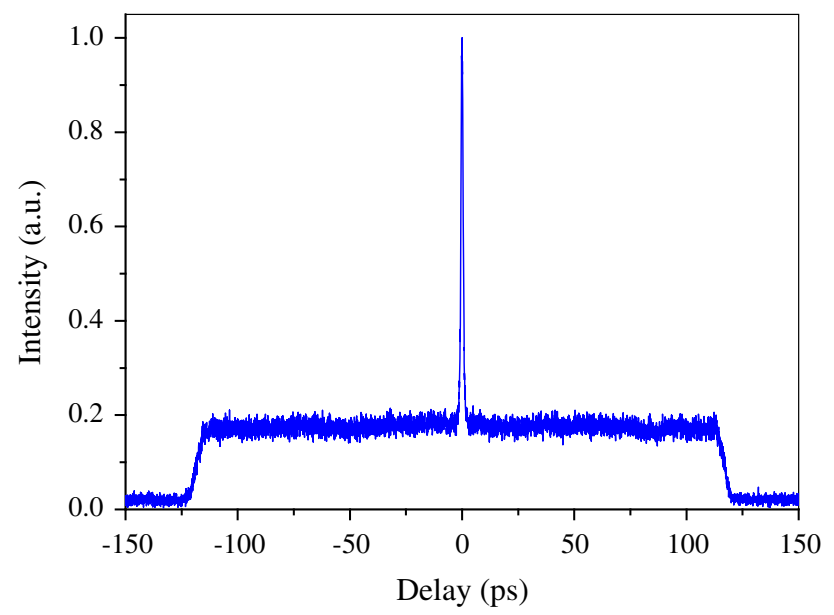

Fig. 4 Autocorrelation trace of a solitons rain

video) illustrates such a phenomena. The corresponding optical spectrum given in Fig. 3 exhibits sidebands. The latter is the result of dispersive waves generated by the solitons during their propagation in the cavity. The absence of modulation in the spectrum proves that the solitons are not bound. The optical autocorrelation trace in Fig. 4 allows us to deduce the pulse duration, we obtain $830 \mathrm{fs}$. The large pedestal confirms that the solitons are in relative motion in the cavity. This behavior is very similar to that reported in [19] in which the authors named this type of dynamic a "soliton rain". However, we have to fully characterize this dynamical behavior before to definitely conclude about the exact soliton dynamics. For that, we have varied the orientation of the polarization controller together with the pumping power.

Let us first consider the adjustment of the polarization controller which allows to vary the nonlinear losses of the cavity. For a pump power of $1.6 \mathrm{~W}$, the results are shown

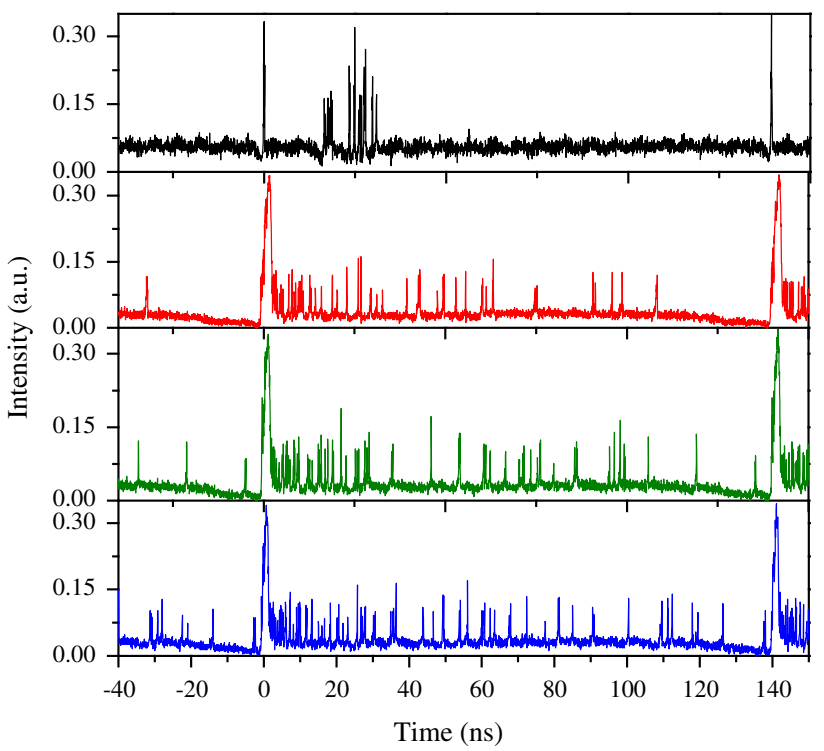

Fig. 5 Temporal evolution of the formation of rains of solitons changing polarization

in Fig. 5 and clearly show that nonlinear losses allow to control the soliton dynamics. The polarization controller has been slightly adjusted. The upper curve illustrates the case where the nonlinear filtering stops low intensities. In such condition, there is no quasi-cw background and no rain of solitons occurs: We observe a classical soliton bunch. While the PC is changed, a significant background appears with soliton bunching and also single solitons. Due to the large number of individual pulses and their high drift velocity, it is not possible to estimate the rate of soliton creation nor the drift velocity with our actual detection apparatus which has intrinsic limitations. By further varying the PC, the background increases and the number of solitons which arises from the background fluctuations to join the condensate phase increases gradually. The size of the condensed phase depends on PC. In addition, the velocity of the isolated solitons is faster than the condensed phase as evidenced by the video. In the case of anomalous dispersion, the central wavelength of the drifting pulses is shorter than the condensed phase. Indeed, the group velocity decreases if the wavelength increases in negative net dispersion. This dynamic corresponds to the one reported in the passively mode-locked fiber laser through NLPR [19]. Indeed, the three elements that characterize the soliton rain are present, i.e., a condensed phase, a continuous background and individual solitons which drift in the cavity.

Figure 6 shows the evolution of the soliton distribution for increasing pumping power for a position of the PC which favors the solitons rain. Results demonstrate that there is a pumping threshold, about $1.4 \mathrm{~W}$, below which 
Fig. 6 Temporal evolution of the formation of rain of solitons for increasing pumping power

Fig. 7 Temporal evolution of the formation of a solitons rain changing polarization controller
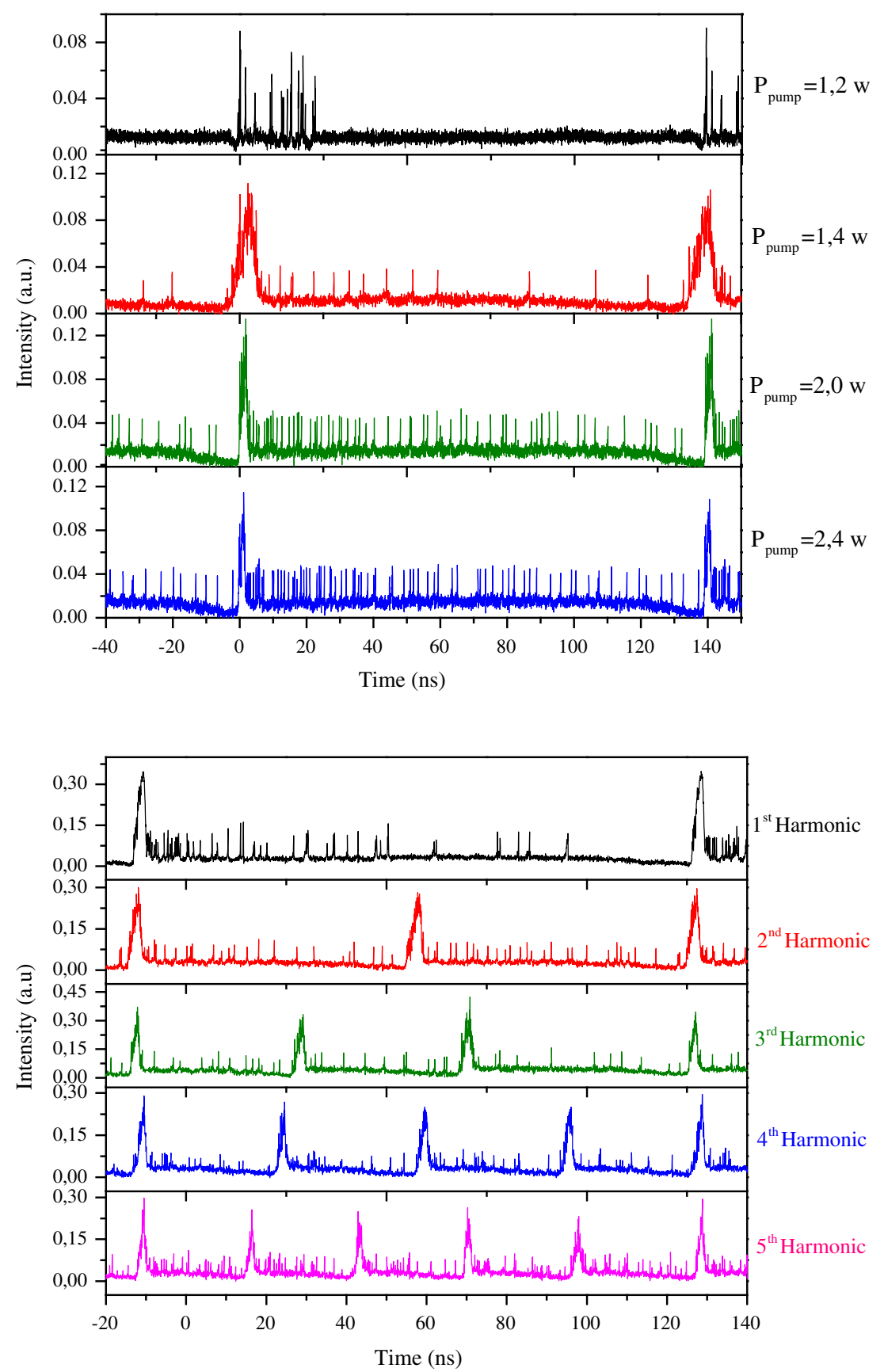

solitons rain does not occur because the level of the background is too low. Alternatively, if the pumping power is too high, the solitons rain is lost and the soliton distribution is a soliton gas [4]. In addition, by varying the pump power from 1.4 to $2.4 \mathrm{~W}$, the number of the individual solitons increases while the temporal width of the condensed phase decreases from about $10 \mathrm{~ns}$ to about 4 ns.

We consider now the situation of lowest curve of Fig. 6 obtained for $P_{\mathrm{p}}=2.4 \mathrm{~W}$. We have then adjusted properly and slightly the orientation of the PC. At first, the number of isolated solitons decreases and then we suddenly obtain several groups of rains of solitons along the cavity. Although these rains are different concerning the size of their condensed phases, the average number of isolated solitons and the background level fluctuations of quasi-cw, these groups represent the harmonics of rain of solitons. Some examples of harmonic mode-locking of solitons rain are shown in Fig. 7. The gradual adjustment of the polarization controller allowed us to pass successively from $1 \mathrm{st}$ to 5 th harmonic. The random formation of solitons on a continuous background has been suggested to be responsible for the differences between the successive periods of solitons rains [19]. 


\section{Conclusion}

In conclusion, we have experimentally observed a rain of solitons in a double-clad laser passively mode-locked through the NOLM, with totally anomalous dispersion. We have studied the dynamics of the rain with the polarization controller and the pumping power. For a fixed pump power, the adjustment of the polarization controller has permitted to pass from 1st to 5th harmonic of rain of solitons per cavity round trip. The observation of this dynamics tends to strengthen the idea of the universality of the collective behavior of dissipative solitons in fiber lasers.

Acknowledgments We thank the Agence Nationale de la Recherche for supporting this work (Contract ANR-2010-BLANC-0417-01SOLICRISTAL).

\section{References}

1. P. Grelu, N. Akhmediev, Dissipative solitons for mode-locked lasers. Nat. Photonics 6, 84-92 (2012)

2. W.H. Renninger, A. Chong, F.W. Wise, Dissipative solitons in normal dispersion fiber lasers. Phys. Rev. A 77, 023814 (2008)

3. H. Zhang, D.Y. Tang, R.J. Knize, L.M. Zhao, Q.L. Bao, K.P. Loh, Graphene mode-locked, wavelength tunable, dissipative soliton fiber laser. Appl. Phys. Lett. 96, 111112 (2010)

4. F. Amrani, A. Haboucha, M. Salhi, H. Leblond, A. Komarov, F. Sanchez, Dissipative solitons compounds in a fiber laser: analogy with the states of the matter. Appl. Phys. B 99, 107-114 (2010)

5. A. Haboucha, H. Leblond, M. Salhi, A. Komarov, F. Sanchez, Analysis of soliton pattern formation in passively mode-locked fiber lasers. Phys. Rev. A 78, 043806 (2008)

6. D.Y. Tang, B. Zhao, L.M. Zhao, H.Y. Tam, Soliton interaction in a fiber laser. Phys. Rev. E 72, 016616 (2005)
7. K. Tamura, H.A. Haus, E.P. Ippen, Self-starting additive pulse mode-locked erbium fibre ring laser. Electron. Lett. 28, 2226-2228 (1992)

8. M. Hofer, M.H. Ober, F. Haberl, M.E. Fermann, Characterization of ultrashort pulse formation in passively mode-locked fiber lasers. IEEE J. Quantum Electron. 28, 720-728 (1992)

9. V.J. Matsas, T.P. Newson, D.J. Richardson, D.N. Payne, Selfstarting passively mode-locked fiber ring soliton laser exploiting nonlinear polarization rotation. Electron. Lett. 28, 1391-1392 (1992)

10. A. Komarov, K. Komarov, D. Meshcheriakov, F. Amrani, F. Sanchez, Polarization dynamics in nonlinear anisotropic fibers. Phys. Rev. A 82, 013813 (2010)

11. I.N. Duling III, Subpicosecond all-fibre erbium laser. Electron. Lett. 27, 544-545 (1991)

12. D.J. Richardson, R.I. Laming, D.N. Payne, M.W. Phillips, V.J. Matsas, $320 \mathrm{fs}$ soliton generation with passively mode-locked erbium fibre laser. Electron. Lett. 27, 730-732 (1991)

13. F. Amrani, M. Salhi, Ph Grelu, H. Leblond, F. Sanchez, Universal soliton pattern formations in passively mode-locked fiber lasers. Opt. Lett. 36, 1545-1547 (2011)

14. F. Amrani, M. Salhi, H. Leblond, F. Sanchez, Characterization of soliton compounds in a passively mode-locked high power fiber laser. Opt. Commun. 283, 5224-5230 (2010)

15. D.Y. Tang, W.S. Man, H.Y. Tam, P.D. Drummond, Observation of bound states of solitons in a passively modelocked fiber laser. Phys. Rev. A 64, 033814 (2001)

16. Ph Grelu, F. Belhache, F. Gutty, J.M. Soto-Crespo, Phase-locked soliton pairs in a stretched-pulse fiber laser. Opt. Lett. 27, 966-968 (2002)

17. N.N. Akhmediev, A. Ankiewicz, J.M. Soto-Crespo, Multisoliton solutions of the complex Ginzburg-Landau equation. Phys. Rev. Lett. 79, 4047-4051 (1997)

18. A. Komarov, K. Komarov, F. Sanchez, Quantization of binding energy of structural solitons in passive mode-locked fiber lasers. Phys. Rev. A 79, 033807 (2009)

19. S. Chouli, Ph Grelu, Soliton rains in a fiber laser: an experimental study. Phys. Rev. A 81, 063829 (2010)

20. C. Bao, X. Xiao, C. Yang, Soliton rains in a normal dispersion fiber laser with dual-filter. Opt. Lett. 38, 1875-1877 (2013) 